\title{
Idiopathic restrictive cardiomyopathy is part of the clinical expression of cardiac troponin I mutations
}

Jens Mogensen, ${ }^{1}$ Toru Kubo, ${ }^{1,2}$ Mauricio Duque, ${ }^{3}$ William Uribe, ${ }^{3}$ Anthony Shaw, ${ }^{1}$ Ross Murphy, ${ }^{1}$

Juan R. Gimeno, ${ }^{1}$ Perry Elliott, ${ }^{1}$ William J. McKenna ${ }^{1}$

${ }^{1}$ Department of Cardiological Sciences, St. George's Hospital Medical School, London, United Kingdom

${ }^{2}$ Departamento de Cardiologia, Clinica Medellin, Medellin, Colombia

${ }^{3}$ Department of Medicine and Geriatrics, Kochi Medical School, Japan

Original citation: J. Clin. Invest. 111:209-216 (2003). doi:10.1172/JCI200316336.

Citation for this corrigendum: J. Clin. Invest. 111:925 (2003). doi:10.1172/JCI200316336C.

The authors wish to correct errors that appeared in the Methods section and throughout the paper. The correct sentences are below. The authors regret the errors.

Mutation analysis of TNNI3 by direct sequencing identified a $87 \mathrm{~A} \rightarrow \mathrm{G}$ nucleotide substitution of exon 8 resulting in an Asp190Gly amino acid substitution that segregated with the disease in the family (maximal two-point lode score: 4.8 ).

Direct sequencing of TNNI3 identified a 93G $\rightarrow$ A nucleotide substitution of exon 8, which resulted in an Arg192His amino acid substitution.

\section{MPDU1 mutations underlie a novel human congenital disorder of glycosylation, designated type If}

Barbara Schenk, ${ }^{1}$ Timo Imbach, ${ }^{2}$ Christian G. Frank, ${ }^{1}$ Claudia E. Grubenmann, ${ }^{2}$ Gerald V. Raymond, ${ }^{3}$ Haggit Hurvitz, ${ }^{4}$ Annick Raas-Rotschild, ${ }^{5}$ Anthony S. Luder, ${ }^{6}$ Jaak Jaeken, ${ }^{7}$ Eric G. Berger, ${ }^{2}$ Gert Matthijs, ${ }^{8}$ Thierry Hennet, ${ }^{2}$ and Markus Aebi ${ }^{1}$

${ }^{1}$ Institute of Microbiology, Swiss Federal Institute of Technology, Zurich, Switzerland

${ }^{2}$ Institute of Physiology, University of Zurich, Switzerland

${ }^{3}$ Kennedy Krieger Institute, Baltimore, Maryland, USA

${ }^{4}$ Department of Pediatrics, Bikur Cholim Hospital, Jerusalem, Israel

${ }^{5}$ Genetic Clinic, Hadassah University Hospital, Jerusalem, Israel

${ }^{6}$ Department of Pediatrics, Sieff Hospital, Safed, Israel, and Faculty of Medicine, Technion, Haifa, Israel

${ }^{7}$ Department of Pediatrics, University Hospital, Leuven, Belgium

${ }^{8}$ Center for Human Genetics, Catholic University, Leuven, Belgium

Original citation: J. Clin. Invest. 108:1687-1695 (2001). doi:10.1172/JCI200113419.

Citation for this corrigendum: J. Clin. Invest. 111:925 (2003). doi:10.1172/JCI200113419C.

During the preparation of this manuscript for publication, errors were introduced into the author list. The corrected author list and affiliations appear below. The authors regret these errors.

Barbara Schenk, ${ }^{1}$ Timo Imbach, ${ }^{2}$ Christian G. Frank, ${ }^{1}$ Claudia E. Grubenmann, ${ }^{2}$ Gerald V. Raymond, ${ }^{3}$ Haggit Hurvitz ${ }^{4}$ Isabelle Korn-Lubetzki, ${ }^{4}$ Shoshana Revel-Vik,${ }^{4}$ Annick Raas-Rotschild, ${ }^{5}$ Anthony S.Luder, ${ }^{6}$ Jaak Jaeken, ${ }^{7}$ Eric G. Berger, ${ }^{2}$ Gert Matthijs, ${ }^{8}$ Thierry Hennet, ${ }^{2}$ and Markus Aebi ${ }^{1}$

${ }^{1}$ Institute of Microbiology, Swiss Federal Institute of Technology, Zurich, Switzerland

${ }^{2}$ Institute of Physiology, University of Zurich, Switzerland

${ }^{3}$ Kennedy Krieger Institute, Baltimore, Maryland, USA

${ }^{4}$ Department of Pediatrics, Bikur Cholim Hospital, Jerusalem, Israel

${ }^{5}$ Genetic Clinic, Hadassah University Hospital, Jerusalem, Israel

${ }^{6}$ Department of Pediatrics, Sieff Hospital, Safed, Israel, and Faculty of Medicine, Technion, Haifa, Israel

${ }^{7}$ Department of Pediatrics, University Hospital, Leuven, Belgium

${ }^{8}$ Center for Human Genetics, Catholic University, Leuven, Belgium 